\title{
The Pathogenesis of Trimethyltin Chloride-Induced Nephrotoxicity
}

\author{
DONALD G. RobERTSON, ${ }^{*} \uparrow$ SANG-NAM KIM, $\uparrow$ RobERT H. GRAY, ${ }^{*}$ \\ AND FELIX A. DE LA IGLESIA* ${ }^{*}$
}

\begin{abstract}
${ }^{*}$ Department of Environmental and Industrial Health, School of Public Health, The University of Michigan, Ann Arbor, Michigan 48109, and $\dagger$ Department of Pathology and Experimental Toxicology, Warner-Lambert/ParkeDavis Pharmaceutical Research, Ann Arbor, Michigan 48105
\end{abstract}

\begin{abstract}
The Pathogenesis of Trimethyltin Chloride-Induced Nephrotoxicity. RoBERTSON, D. G., KIM, S. N., GRAY, R. H., AND DE LA IGLESIA, F. A. (1987). Fundam. Appl. Toxicol. 8, 147-158. In exploratory studies aimed at elucidating CNS effects due to heavy metal toxicity, signs of compromised renal function were seen in rats. The studies reported here describe the sequential steps of the development of nephrotoxicity by trimethyltin chloride (TMT) in rats. Single doses of $12.25 \mathrm{mg} / \mathrm{kg}$ TMT administered orally to 150 - to $175 . \mathrm{g}$ Long-Evans rats elicited overt signs of toxicity including behavioral abnormalities and marked weight loss. Concurrent with the development of these signs, nephrotoxicity was manifested as functional kidney compromise and associated histopathologic evidence of tubular damage. Pathological changes in the kidneys from treated rats were hyaline droplet inclusions, attenuated brush border, basolateral vacuolization, and eosinophilic granular casts in the proximal tubule cells. These lesions were detected as early as 2 days post-treatment and progressed with time in an orderly and sequential fashion. Renal lesions between 5 and 8 days were mild to severe cortical tubular dilatation, hydropic degeneration, and diffuse hyaline droplet deposition in the lower nephron tubules. Medullary edema and exfoliation of degenerated tubular epithelial cells with cast formation followed from 8 to 11 days. The morphological changes were accompanied by marked elevation of blood urea nitrogen, parallel with polyuria at Day 2 and oliguria by Day 14. Behavioral abnormalities as well as weight loss correlated well with the time course and severity of renal dysfunction and progression of morphological changes. A second experiment compared the effects of TMT in rats of different weights. Heavier rats were more sensitive than lighter rats to the nephrotoxic effects of TMT. These effects were independent of recognizable neurotoxic effects of TMT in the hippocampus. (1) 1987 Society of Toxicology.
\end{abstract}

Trimethyltin (TMT) is a member of the organotin class of compounds used widely in industry as plasticizers of polyvinyl chloride products (Evans, 1974) and as biocides incorporated in molluscicides, insecticides, fungicides, and bactericides (Piver, 1973). TMT induces focal pathologic lesions of the brain, preferentially in the hippocampus and other limbic system structures (Brown et al., 1979). However, in the time course of events leading to CNS damage, accumulated evidence indicates that early lesions also develop in the kidney (Robertson et al., 1984; Opacka and Sparrow, 1985).
Although the neurotoxic effects of TMT have been well documented, the nephrotoxic effects of the compound have received little attention. Brown et al. (1979) reported hydronephrosis and vacuolar degeneration of renal tubules in rats administered oral doses of $4 \mathrm{mg} / \mathrm{kg}$ at weekly intervals. However, Bouldin et al. (1981) reported no effects in the kidney of adult and neonatal rats administered TMT in either acute or chronic dosing regimens. Brown et al. (1984) reported no evidence of nephrotoxicity in marmosets receiving TMT in various dosing regimens. Preliminary studies in our laboratories indi- 
cated that TMT increased blood urea nitrogen (BUN) levels in young adult rats and produced moderate tubular dilatation and epithelial vacuolization (Robertson et al., 1984). In addition, there was evidence of a parallel time-course relationship between the effects on the kidney and various behavioral manifestations of TMT intoxication mimicking the "TMT syndrome" as described by Dyer et al. (1982a). Opacka and Sparrow (1985) recently documented the nephrotoxic effects on rats exposed to acute oral doses of 3,6 , and $10 \mathrm{mg} / \mathrm{kg}$ TMT. These effects were evident as early as $3 \mathrm{hr}$ after dosing and were characterized by marked tubular damage, increased urinary $\mathrm{pH}$, and polyuria.

TMT-induced neurotoxicity has been suggested as an experimental model for the study of various neurological deficits including epilepsy (Dyer et al., 1982b), limbic system dysfunction (Dyer et al., 1982a), learning and memory disorders (Walsh et al., 1982), minimal brain dysfunction (Swartzwelder et al., 1983), and hyperkinetic syndrome (Swartzwelder et al., 1983). With this increased interest in the neurotoxicity mechanisms of TMT, it is critical that interrelationships of TMT with other organs systems be investigated. Thus, the time course and extent of TMT-induced nephrotoxicity is essential to determine whether this nephrotoxicity correlates with the development of recognizable neurotoxic manifestations. Dyer et al. (1982a) demonstrated that heavy rats were particularly sensitive to the acute toxicity of TMT. In addition to the straightforward nephrotoxicity study, we wished to determine whether differences in the extent of kidney pathology can also be attributed to weight (possibly age) differences in rats.

\section{METHODS}

Animals and housing. Male Long-Evans rats obtained from Charles River Laboratories were housed in single sanitized cages in air-conditioned quarters. Water and food were offered ad libitum and the consumption of each was measured by differential weight every third day. Animals were maintained in a 12 -hr photoperiod.

Experiment $I$. In the first experiment, 43 adult rats were dosed po by single administration of $12.25 \mathrm{mg} / \mathrm{kg}$ of TMT chloride (K\&K Laburatories) dissolved in distilled water. Preliminary studies determined this level to be equivalent to the 14-day median lethal dose in this species, in good agreement with the level $(12.6 \mathrm{mg} / \mathrm{kg})$ determined by Brown et al. (1979). TMT is a potent neurotoxicant with a significant vapor pressure. Handling of the compound, including dosing, was done in a vertical laminar flow hood or with appropriate respiratory protection. In addition, lab coats, double thickness latex gloves, and safety goggles were worn for protection of the skin and eyes. Fifteen rats were given distilled water and served as controls. All animals were observed daily for clinical signs of toxicity and weighed every third day. Sacrifice order was determined by a computerized random number generator before the experiment was initiated. If an animal died before its scheduled sacrifice, the rat with the next number on the list was used to replace it. Permanent identification numbers were assigned at sacrifice. Five treated animals were taken randomly at 2, 5, 8, 11 , and 14 days after dosing, sacrificed, and examined for gross pathologic and microscopic lesions. Three control animals were sacrificed along with each treated group. Predose weights of control and treated rats were $234 \pm 1.9 \mathrm{~g}$ $(n=15)$ and $232 \pm 1.2 \mathrm{~g}(n=43)$, respectively.

Urines (24 hr) were collected on Days 2, 8, and 14. Total volume and specific gravity of urine were determined. Urine protein was measured semiquantitatively by reagent strip and quantitatively by sulfosalicylic precipitation methods. Blood, bilirubin, glucose, and ketones were measured semiquantitatively on an automated reagent strip reader (Clinitek). At sacrifice, animals were anesthetized by ether inhalation and blood was obtained by cardiac puncture. Kidneys were examined for gross changes, sliced, placed in 10\% neutralbuffered formalin, and processed for paraffin embedding. Sections were cut and stained with PAS and hematoxylin-eosin. Frozen sections were stained with oil red-O for evaluation of fat deposition. For the purpose of evaluating nuclear density changes attributable to cell loss or necrosis, the cortex and medulla of kidneys were evaluated quantitatively with a semiautomated procedure using Feulgen-stained sections according to a method described elsewhere (Weinberg et al., 1987).

Serum BUN levels were determined using the colorimetric urease technique (Behring Diagnostics) in a RotoChem clinical chemistry unit. Creatinine levels were determined using a sodium picrate colorimetric technique (Worthington) in the same unit.

Experiment II. Three groups of six rats each in three different weight classes were used: heavy ( 450 to $525 \mathrm{~g}$ )-, medium ( 300 to $400 \mathrm{~g}$ )-, and light-weight rats (150 to 250 g). Three rats in each group were dosed orally with $3 \mathrm{mg} /$ kg TMT for 3 consecutive days. The remaining three rats were given water and served as controls. 


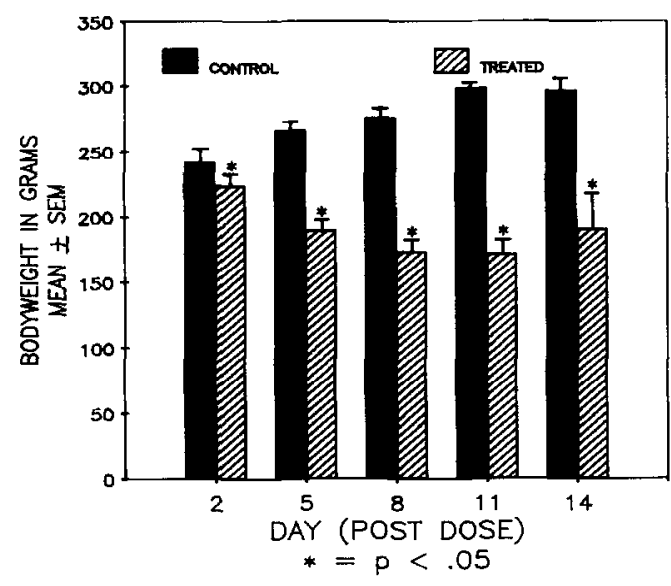

FIG. 1. Weight changes in relation to time after TMT administration. Each bar represents the mean weight of surviving animals in each group; treated animals received a single dose of $12.25 \mathrm{mg} / \mathrm{kg}$. $N$ for each time point is as follows (control/treated): Day 2 (15/43), Day 5 (12/ $38)$, Day $8(9 / 26)$, Day $11(6 / 13)$, and Day $14(3 / 7)$.

Animals were observed and weighed daily. Moribund animals were bled by orbital puncture for blood biochemical determinations and then sacrificed. The surviving animals were sacrificed 14 days after dosing. At sacrifice rats were anesthetized with ether and perfused through the heart with ice-cold physiological saline for 3 min followed by ice-cold $10 \%$ neutral-buffered formalin for $10 \mathrm{~min}$ with the descending aorta clamped to prevent perfusion of the kidneys. Brain and kidneys were further fixed in $10 \%$ formalin overnight and processed for paraffin embedding. Kidney sections were stained with the PAS-hematoxylin-eosin and brain sections with ironhematoxylin. BUN levels were determined as described above.

Numerical data for blood BUN levels and animal weights after TMT treatment were expressed as percentage of control values and as the means $\pm S E$ of the means, respectively. Student's two-tailed $t$ test was used to establish differences between group means with $p<0.05$ as the significance level.

\section{RESULTS}

Experiment 1. Of 43 treated animals in this experiment, 8 animals were found dead and 8 animals were sacrificed in moribund condition. Of these 16 animals, 7 died within 8 days postdose. The first dead animal was found 5 days after dosing and the last one on Day 13. The time course and extent of weight loss are indicated in Fig. 1. Treated animals showed significant decreases in body weight as compared with control animals at every time point. After initial weight loss, no weight gains were observed in treated animals on Days 2 and 5. On Day 8, 15\% (4 of 26) of all treated animals gained weight as compared with the previous weighing. On Day $11,38 \%$ (5 of 13) of treated animals and, on Day 14, $58 \%$ ( 4 of 7 ) had weight gains. The mean weight of treated animals on Day 14 was higher than on Day 11.

During the experiment, food consumption was significantly decreased compared with that of control animals, with control animals consuming an average of $22.6 \mathrm{~g} /$ day and treated animals consuming an average of $12.5 \mathrm{~g} /$ day. Water consumption was not significantly different between control and treated animals, 35.4 and $34.9 \mathrm{ml} /$ day, respectively.

Marked behavioral changes were observed in treated animals. The first sign noted was persistent head tremors which gradually progressed to generalized body tremors. Animals were hyperactive and hyperreactive upon handling and occasional spontaneous motor seizures were observed. Preceding death, animals became ataxic, with eventual prostration and stupor. Onset of tremors was as early as $24 \mathrm{hr}$ after dose in 2 of 43 animals. Maximal behavioral changes were evident on Days 5-8 with $95 \%$ of surviving animals exhibiting behavioral abnormalities. Of 43 animals, 10 had spontaneous motor seizures with the first occurrence 4 days after dosing and occurrences still evident up to 11 days after dosing. Behavioral abnormalities gradually subsided in severity and frequency after 9 days.

Urine volumes $(24 \mathrm{hr})$ were significantly increased for treated animals on Day 2 (Table 1). However, specific gravity was not decreased. Total urine protein excretion in 24hr samples was significantly elevated on Day 2. On Day 14, urine volume was significantly decreased as was total urine protein. No changes were seen in urine ketones or bilirubin. Four animals on Day 8 and 3 animals on Day 14 had moderate to marked occult blood 


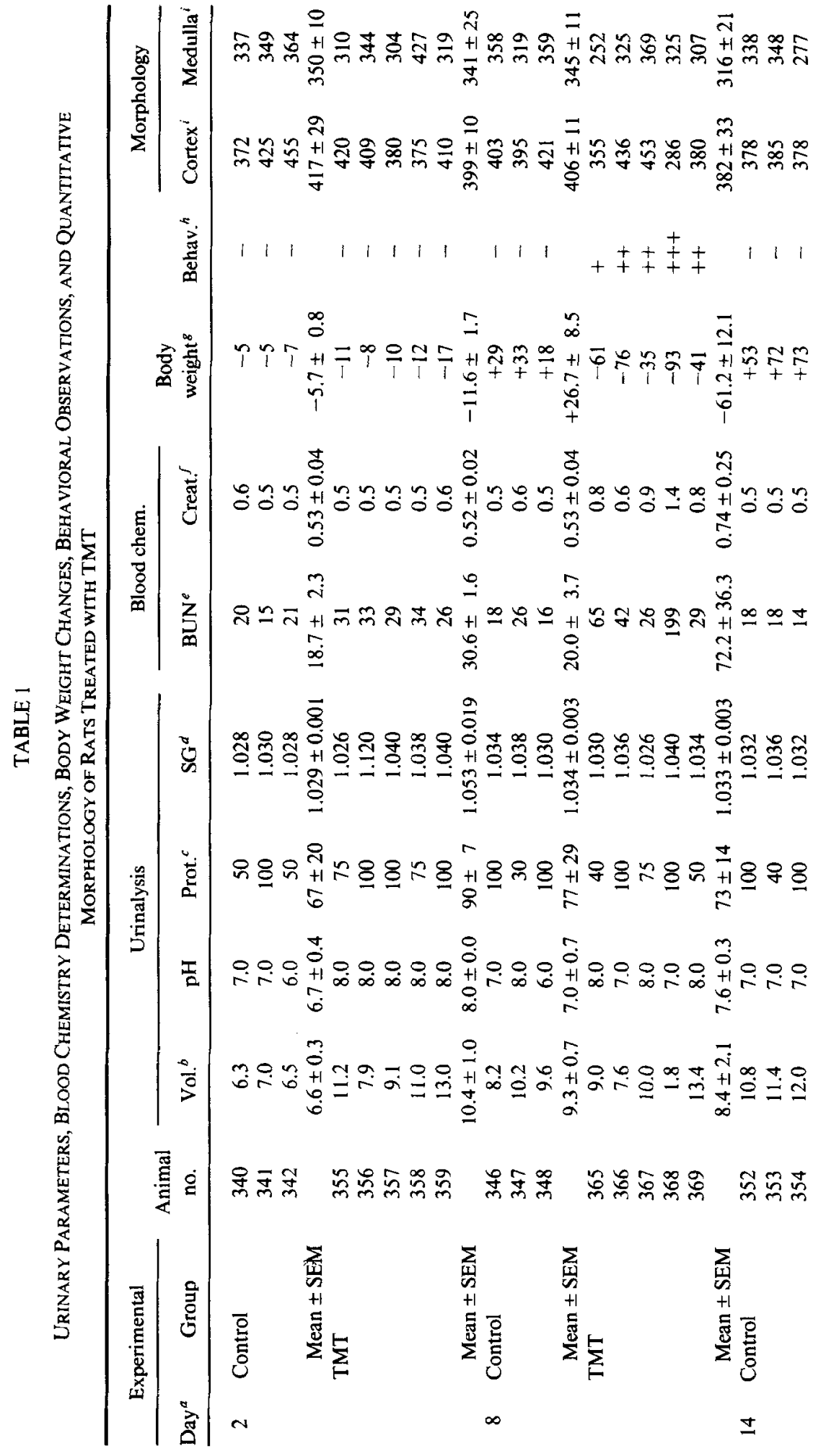




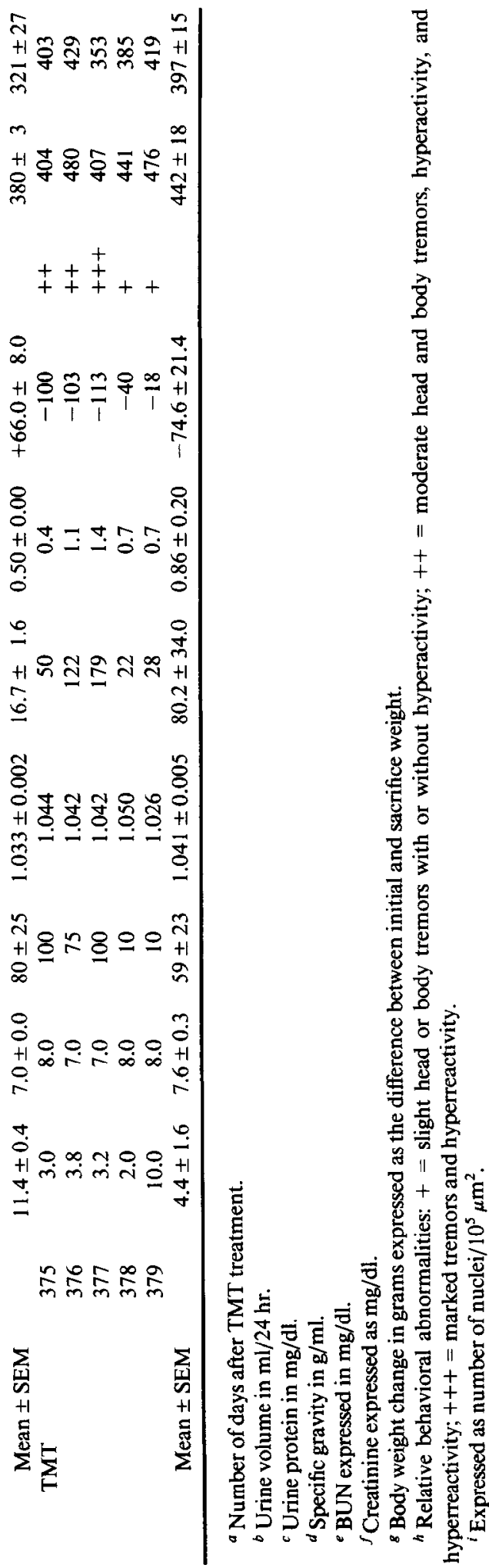

in urine. The $\mathrm{pH}$ of the urine was significantly elevated on Day $2(\mathrm{pH} 8.0)$ as compared with $\mathrm{pH} 6.7$ for controls and was above controls on all subsequent days.

The time course and extent of BUN changes are indicated in Fig. 2. BUN levels were progressively increased during the course of the study, reaching a group mean of $80 \mathrm{mg} / \mathrm{dl}$ by Day 14 . On each of Days 8,11 , and 14,2 of 5 treated animals had BUN levels under $29 \mathrm{mg} / \mathrm{dl}$ while the remaining animals had higher BUN levels. Rats with lower BUN levels invariably showed weight gain. When the data of all animals that gained weight (recovery data in Fig. 2) were separated from those of animals which did not gain weight, all BUN increases in nonrecovering animals were statistically significant at Days 8,11 , and 14. Moribund animals were followed closely and, when treated as a group, had a mean BUN level of $150 \mathrm{mg} / \mathrm{dl}$ with values ranging from 71 to $243 \mathrm{mg} / \mathrm{dl}$.

Creatinine levels were moderately increased; however, statistical significance was not established. The highest creatinine level observed was $1.4 \mathrm{mg} / \mathrm{dl}$ with four treated animals having levels over $1 \mathrm{mg} / \mathrm{dl}$.

Upon gross pathological examination, kidneys from moribund animals were pale or mottled. No gross changes were seen in other sacrificed animals. Figure 3a illustrates the earliest pathologic changes in proximal tubules observed 2 days after dosing, including the cortical area (Fig. 3b). The proximal tubules in the inner cortex were dilated with attenuated brush borders and often contained light eosinophilic casts in the lumina (Fig. 3c). PAS-positive hyalin droplets were seen in the cytoplasm of proximal convoluted tubules and collecting ducts. Hyalin droplets were later observed in the pars recta of the tubules, in collecting ducts, in the thin loop of Henle, and in the pelvic urothelium. The straight tubules of the pars recta showed diffuse hydropic degeneration with pyknotic nuclei in addition to hyalin droplets as early as Day 5 (Figs. 3d,e). Many mitotic figures were seen in the straight tubules and occa- 


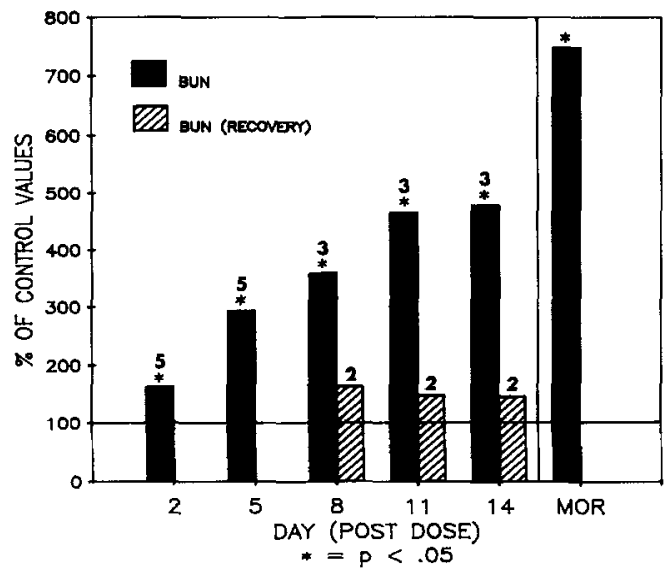

FIG. 2. Effects of TMT on blood BUN levels after TMT treatment. Each bar represents the mean BUN levels expressed as percentage of control values (average of three values) at each postdose interval. BUN (recovery) indicates the mean BUN level obtained for treated animals that gained weight on a given day. MOR = moribund animals ( $n-8)$, compared with Day 14 control values but not analyzed statistically. The number above each bar indicates $n$ for that measurement.

sionally in the collecting ducts in the inner medulla (Fig. 3f). The morphometric evaluation of the kidneys showed no consistent pattern of cellular density change following TMT treatment (Table 1).

Kidneys of rats sacrificed on Day 8 and thereafter showed increased hyalin droplet inclusions in the straight tubules, collecting ducts, and thin loop of Henle as well as in interstitial macrophages (Fig. 4a). The epithelial lining of the collecting ducts was laden with hyalin droplets (Fig. 4b) and was occasionally detached from the basement membrane and exfoliated into the lumen. The tubules in the outer and inner medulla were diffusely edematous and partially obliterated the tubular lumen, encroaching upon peritubular vessels. The distal convoluted tubules often showed hydropic degeneration. The transitional epithelium at the level of the area of cribosa was swollen and contained numerous hyalin droplets (Fig. 4c). The severity of renal lesions was progressive and was most prominent between Days 8 and 14 (Figs. 4df). At termination several animals showed milder tubular lesions indicative of a recovery process, while others had persisting severe lesions.

Experiment II. Two of three treated heavy animals were sacrificed in extremis on Days 3 and 7 , respectively, and the remaining animal died on Day 6. No mortality was observed in any other group. The pattern of weight loss is indicated in Fig. 5. Treated heavy animals lost an average of $153 \mathrm{~g}$ while corresponding control animals gained $6 \mathrm{~g}$ during the study. Intermcdiate-wcight animals initially lost weight and by Day 5 had lost about $32 \mathrm{~g}$. After Day 5 , animals progressively recovered weight and by Day 14 averaged 11 -g gains. Light-weight rats did not lose weight but had significant decreases in weight gain as compared with control animals.

Heavy-weight animals exhibited marked behavioral abnormalities within $24 \mathrm{hr}$ of dosing, consisting of slight to moderate head tremors and progressing to generalized body tremors. Tail mutilation was also observed. Death was preceded by hyperactivity, hyperreactivity, abnormal gait, ataxia, prostration, and stupor. No tail mutilation was seen in the animals of the other groups. The two treated rats from the heavy-weight group, sacrificed in extremis, had BUN levels of 140 and 180 $\mathrm{mg} / \mathrm{dl}$.

Pathologic changes of the kidneys from heavy treated rats were similar to those as described for animals in experiment I. Marked damage to the tubules in the pars recta, marked hyalin droplet inclusions, and many mitotic figures were observed in all treated heavy animals. Surprisingly, light- and medium-weight rats showed no evidence of kidney damage on Day 14.

The hippocampus of heavy rats (all of which were sacrificed by Day 7) showed only mild cellular thinning in the dorsal pyramidal cell layers with no evidence of pyknosis. The light and medium rats (sacrificed on Day 14) displayed marked CAl pyramidal cell loss. This loss was most pronounced in the dorsal (septal) hippocampus. Cellular loss was also evident in the CA3-4 pyramidal cell region; 

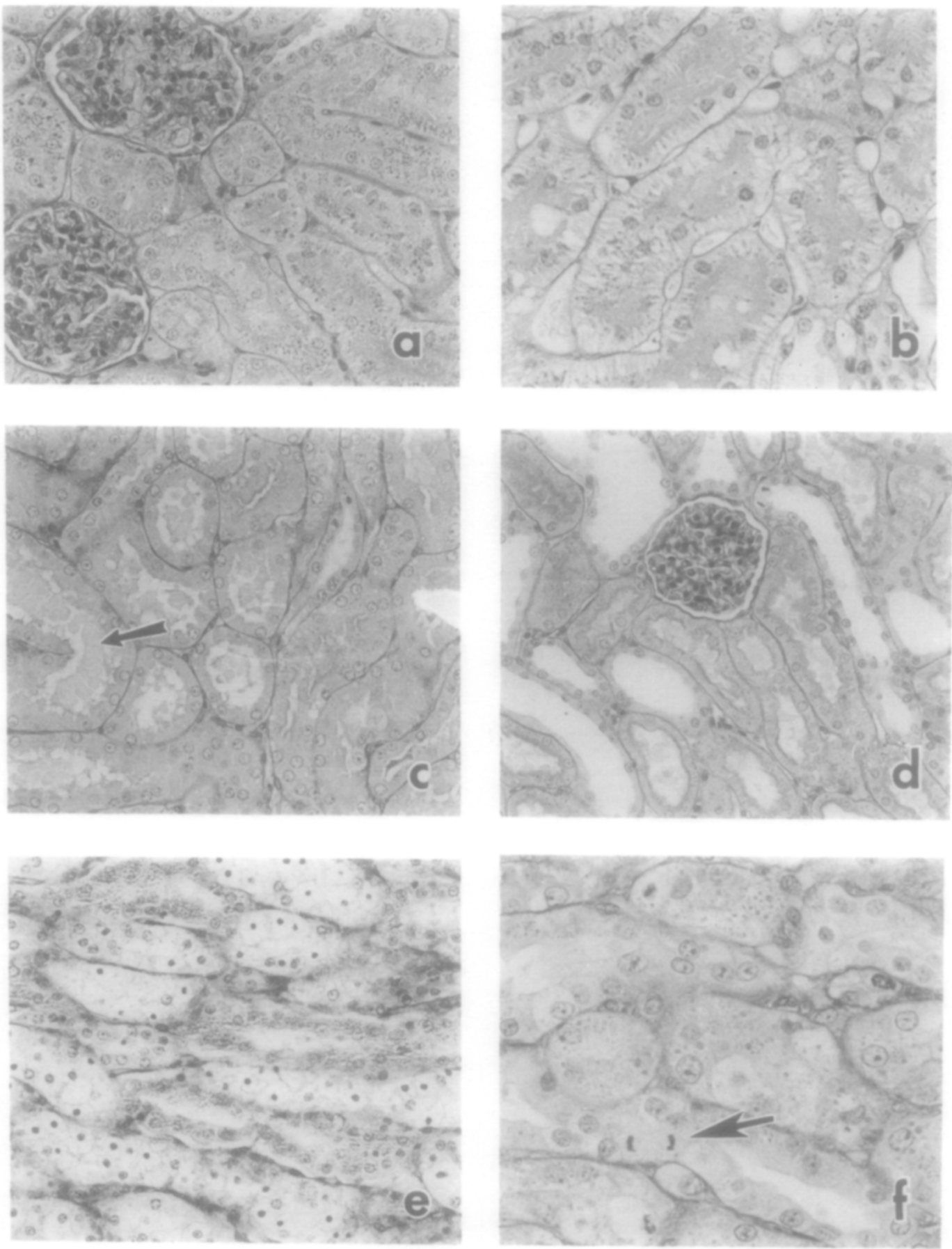

FIG. 3. (a-c) Kidney cortex of rats 2 days after single dose of $12.25 \mathrm{mg} / \mathrm{kg}$ TMT. (a) Glomeruli appear normal and the proximal tubules contain cytoplasmic hyaline droplets. $\times 250$. (b) Proximal tubule cells in the cortex with basolateral vacuolation. $\times 400$. (c) Inner cortex tubular cells have attenuated brush borders. Note the eosinophilic granular casts in the lumen (arrow). $\times 250$. (d-f) Cortex of rat kidney after 5 to 7 days TMT. (d) Prominent tubular dilatation in distal tubules; proximal tubules with attenuated brush borders and with granular casts in the lumen. $\times 200$. (e) Tubules from pars recta with pyknotic nuclei and PAS-positive droplets. $\times 200$. (f) Mitotic figures (arrow) and cytoplasmic inclusions are evident in edematous tubule cells of the pars recta. $\times 400$. 

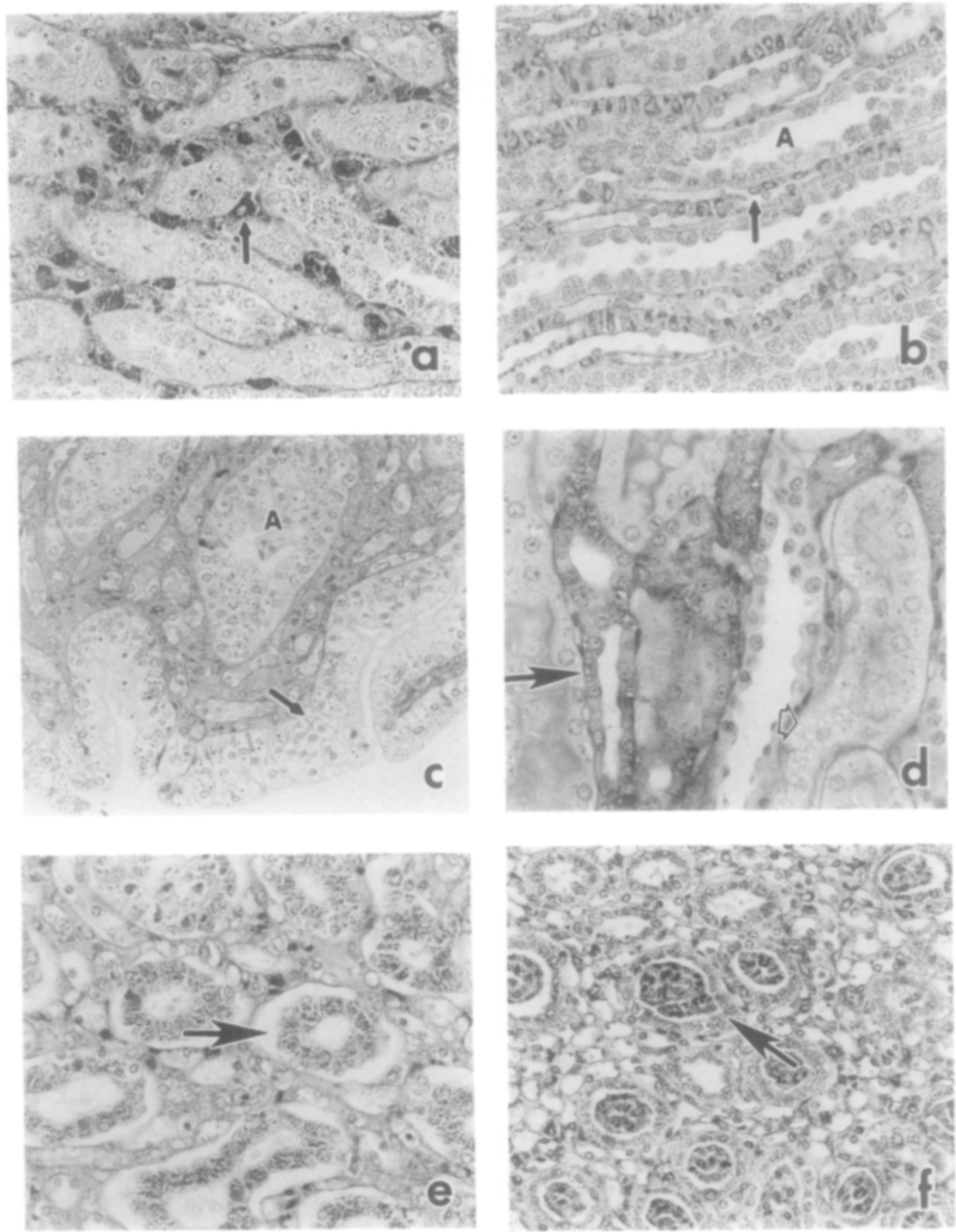

FIG. 4. (a-f) Kidney sections from rats 8 to 14 days after TMT treatment. (a) Tubules in the pars recta with PAS-positive droplets; interstitial cells show inclusions (arrow). $\times 250$. (b) Collecting tubules in the inner medulla with inclusions (A); thin loop of Henle cells also show inclusions (arrow). $\times 250$. (c) Papillary ducts with narrowed lumens and lined with edematous cells (A); swollen transitional epithelium cells at the area cribosa (arrow). $\times 250$. (d) Atrophic distal tubular cells contain PAS-positive droplets (solid arrow); proximal tubular cells show cytoplasmic vacuoles (open arrows). $\times 400$. (e) Detached tubular lining epithelial cells with cytoplasmic inclusions. $\times 400$. (f) Epithelial cell casts in the papillary collecting ducts (arrow). $\times 250$. 


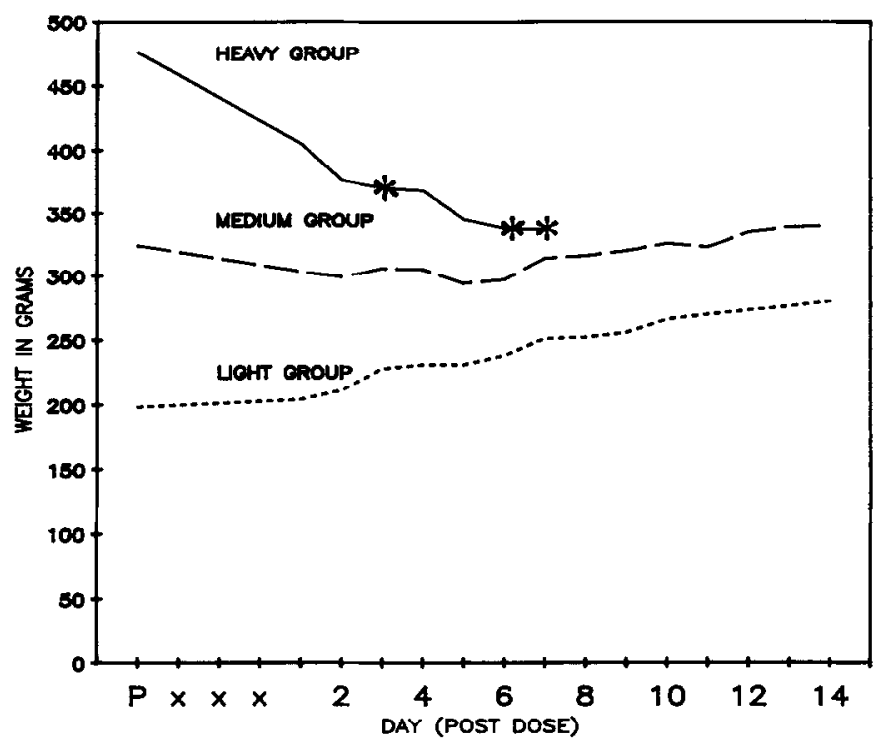

FIG. 5. Changes in body weight in different age-grouped animals due to TMT administration. Heavy group: 450-525 g; medium group: $300-400 \mathrm{~g}$; light group: $150-250 \mathrm{~g}$. There were three control and three treated rats in each weight group. P: predose weight; $\mathrm{X}$ : day of dose administration; *indicates death of an animal. Average weights (in grams) for control rats (predose/sacrifice) were heavy group (503/514), medium group (344/383), and light group (189/291).

however, the damage was not as severe as in the CAl region. Granule cells appeared to be unnaffected.

\section{DISCUSSION}

The results of this study indicate that TMT is a potent nephrotoxicant in the rat, a finding that has been largely undetected in spite of recent emphasis on the study of heavy metal intoxications (Brown et al., 1979; Dyer et al., 1982a). The pathologic changes in the kidney with the supporting clinical laboratory data suggest that TMT-induced renal failure was a contributing factor to death or moribundity in treated animals. Our data are in good agreement with those of Opacka and Sparrow (1985). One exception we noted is the lack of change in water consumption reported in their study. The peak behavioral alterations were seen between Days 5 and 8 and coincided with the maximum extent of renal damage. In a preliminary study (Robertson $e t$ al., 1984) we established the time course of BUN changes in 200- to 250-g rats treated with $3 \mathrm{mg} / \mathrm{kg}$ TMT for 3 consecutive days. Peak BUN levels were found 7 days postdose and followed by normal values at Day 14 . In experiment I, a higher dose was used which resulted in higher BUN levels leading to acute renal failure and death.

The results of the urinalyses suggest that TMT initially induces polyuria and then an oliguric phase as the renal lesion progresses into frank acute renal failure. The polyuric phase was not a typical diuretic effect in that specific gravity did not decrease. Several animals within the experimental group showed marked proteinuria, an indication of marked tubular damage, although these protein increases were not significant on a group basis. Total urine protein was increased on Day 2 and decreased on Day 14 due to changes in total urine volume. The increase in $\mathrm{pH}$ noted in treated animals on Day 2 indicates an inability to acidify urine as one of the earliest effects of TMT. 
One the most important clinical laboratory findings of this study was the marked increase in BUN. Water consumption was not reduced in treated animals, indicating that BUN elevation was not due to dehydration but to marked renal impairment. These elevated BUN levels in moribund animals correlated with the morphological changes in the kidney and indicated clearly that the cause of morbidity was acute renal failure. The wide variability of BUN levels noted on Days 8, 11 , and 14 was attributed to the recovering animals with lower BUN levels sampled together with rats in end-stage renal failure with extremely high BUN levels.

The histogenesis of the renal lesion indicates an initial focal effect of TMT on the tubules in the outer medullary area of kidneys. The hyalin droplets noted in tubular epithelial cells represent the resorption of protein from the glomerular filtrate (Heptinstall, 1966). The overloading of protein in the tubules and subsequent transfer into the interstitium led to the expansion of this compartment, resulting in the obstruction of vascular supply and swelling in the papilla. This interstitial swelling along with tubular blockage caused by epithelial casts may contribute to decreased glomerular filtration rate, oliguria, and uremia. There was no overt evidence of glomerular pathology, suggesting that in the pathogenesis of TMT-induced uremia in rats, the tubular lesions are more important than glomerular dysfunction. However, in-depth testing would be required to preclude the presence of glomerular dysfunction. Mori et al. (1984) observed similar tubular damage in guinea pigs exposed to tributyltin. The doses employed were higher ( 10 and $40 \mathrm{mg} / \mathrm{kg} /$ day for 50 days percutaneously) than those used in our study. Trimethyltin, however, is substantially more hydrophilic than tributyltin, perhaps explaining the former compound's propensity for early profound changes in the kidney. Furthermore, the authors saw no change in glomerular morphology, and creatine clearance was unaffected. These observations are in good agreement with ours, sug- gesting the possibility of a common mechanism in alkyltin-induced kidney damage.

The morphometric studies on the nuclei in the cortex and medulla of rats treated with TMT did not reveal significant density changes from control values. Considerable variation was observed in control and treated animals. There was a trend of increased numbers of nuclei in the treated rats after 14 days, which may reflect hyperplasia in response to the loss or damage of cells as a result of TMT exposure.

Histologic studies of rat renal damage suggest prompt repair and recovery from the nephrotoxic effects of TMT. Mitotic figures were frequent in tubular cells, indicative of an active repair process. The time course of the renal lesions indicates maximal severity at 7 to 11 days following a single oral dose of TMT, and the lesion is at least partially reversible.

The experimental approach used in this study did not allow for elucidation of the mechanism of TMT-induced nephrotoxicity. However, the morphological observations do suggest some possibilities. The overloading of hyalin droplets in tubular epithelial cells suggests that tubular dysfunction was due to altered intracellular processes of metabolism and transport, attributable to impaired energy production. TMT has pronounced effects on oxidative phosphorylation (Aldridge and Street, 1971) which may contribute directly to a decrease in tubular function. Mercuric chloride causes similar damage to the pars recta (McDowell et al., 1976), suggesting that this region may be exquisitely sensitive to metal toxicity.

An important landmark of TMT toxicity is the development of significant behavioral alterations. The scope and extent of these behavioral changes were similar to those described earlier as sequelae of TMT intoxication (Brown et al., 1979; Dyer et al., 1982a). Many of the characteristic behavioral changes seen in the TMT syndrome including tremors, irritability, hyperreactivity, anorexia, hypothermia, seizures, and stupor are 
also seen in patients with kidney failure or uremia due to other causes (Schoenfeld and Humphreys, 1976). This suggests that many aspects of the TMT syndrome previously attributed to a direct neurotoxicity of the compound may actually represent secondary effects of kidney injury and subsequent uremic state. Essman $(1960,1965)$ found increased error scores in methemoglobin-induced uremic rats trained in a $\mathrm{T}$ maze, measured up to 2 weeks after clinical recovery. The onset of behavioral changes observed in our study paralleled the time course of development of nephrotoxicity. Coincident with the improvement of kidney structure and function, there was a gradual amelioration of behavioral abnormalities. This might explain why many signs of the TMT syndrome disappear with time (Bouldin et al., 1981; Dyer et al., 1982a). However, several behavioral deficits in rats treated with TMT persist and do not subside with time, including several central nervous system dysfunctions (Swartzwelder et al., 1981; Walsh et al., 1982; Ruppert et al., 1982; Johnson et al., 1984). Thus, this partially reversible TMT nephrotoxicity does not account for the persistent neurotoxic manifestations.

An important finding in the second experiment was that heavy animals had little CNS damage in the hippocampus pyramidal cells and yet exhibited marked behavioral abnormalities. By contrast, light- and middleweight animals did not display similarly marked behavioral abnormalities although they had pronounced loss of CAl pyramidal cells by Day 14 . Therefore, the immediate behavioral deficits of the TMT syndrome are closely correlated with the development of kidney damage, and were not dependent on overt hippocampal disruption.

In conclusion, our studies demonstrate that TMT is a potent nephrotoxicant and that the nephrotoxicity is dose and weight (possibly age) dependent. The kidney lesion is characterized by marked morphological disruption within the tubules, particularly in the pars recta and medulla, and is accompanied by elevated BUN levels. The time course of the renal lesion indicates maximal severity at approximately 7 to 11 days, and the lesion is at least partially reversible. The nephrotoxicity might be involved in the production of behavioral deficits; however, the TMT-induced hippocampal lesion is independent of the renal lesion.

\section{ACKNOWLEDGMENT}

This work was supported by NIEHS Environmental Toxicology Training Grant ES 07062 (Dr. Robertson).

\section{REFERENCES}

ALDRIDGE, W. N., AND STREET, B. W. (1971). Oxidative phosphorylation-The relation between the specific binding of trimethyltin and triethyltin to mitochondria and their effects on various mitochondrial functions. Biochem. J. 124, 221-234.

ARIEFF, A. I. (1981). Neurological complications of uremia. In The Kidney (B. M. Brenner and F. C. Rector, Eds.), Vol. II, 2nd ed. pp. 2306-2344. Saunders, Philadelphia.

Bouldin, T. W., GoINes, N. D., BAGNell, C. R., AND KrigmaN, M. R. (1981). Pathogenesis of trimethyltin neuronal toxicity-Ultrastructural and cytochemical observations. Amer. J. Pathol. 104, 237-249.

Brown, A. W., AldRIDGe, W. N., StreEt, B. W., AND VERSCHOYLE, R. D. (1979). The behavioral and neuropathologic sequelae of intoxication by trimethyltin compounds in the rat. Amer. J. Pathol. 97, 59-82.

Brown, A. W., Verschoyle, R. D., Street, B. W., AND ALDRIDGE, W. N. (1984). The neurotoxicity of trimethyltin chloride in hamsters, gerbils and marmosets. J. Appl. Toxicol. 4(1), 12-21.

DYer, R. S., WALSII, T. J., WONDERLIN, W. F., AND BERCEGEAY, M. (1982a). The trimethyltin syndrome in rats. Neurobehav. Toxicol. Teratol. 4, 127-133.

DYFR, R. S., WONDERLIN, W. F., AND WALSH, T. J. (1982b). Increased seizure susceptibility following trimethyltin administration in rats, Neurobehav. Toxicol. Teratol. 4, 203-208.

ESSMAN, W. B. (1960). Correlates of behavioral deficits with an experimental induction of acute renal failure in rats. Clin. Res. $8,175$.

ESSMAN, W. B. (1965). Behavioral bioassay for experimentally induced uremic endotoxemia in rats. Perceptual Motor Skills 20, 115-120.

EvaNS, C. J. (1974). Developments in the organotin industry. Tin Research Institute, Publ. No. 491. 
HePtinstall, R. H. (1966). Pathology of the Kidney, Vol. II, 2nd ed. Little, Brown, Boston.

Johnson, C. T., DunN, A., Robinson, C., Walsh, T. J., AND SWARTZWELDER, H. S. (1984). Alterations in regulatory and locomotor behaviors following trimethyltin exposure in the rat: A time and dose analysis. Neurosci. Lett. 47, 99-106.

MCDowell, E. M., NAgle, R. B., Zalme, R. C., MCNeil, J. S., Flamenbaum, W., and Trump, B. F. (1976). Studies on the pathophysiology of acute renal failure. I. Correlation of ultrastructure and function in the proximal tubule of the rat following administration of mercuric chloride. Virchows Arch B Cell Pathol. 22 , 173-196.

MORI, Y., IESATO, K., UEDA, S., MORI, T., OHNISHI, K., SeINo, Y., WaKashin, Y., WaKaShin, M., AND OKUDA, K. (1984). Renal tubular disturbances induced by tributyl-tin oxide in guinea pigs: A secondary Fanconi syndrome. Clin. Nephrol. 21, 118-125.

OPACKA, J., AND SPARROW, S. (1985). Nephrotoxic effect of trimethyltin in rats. Toxicol. Lett. 27,97-102.

PIVER, W. T. (1973). Organotin compounds: Industrial applications and biological investigation. Environ. Health Perspect. 4, 61-79.

RoberTSON, D. G., KIM, S. N., GRAY, R. H., AND DE LA IGLESIA, F. A. (1984). Renal toxicity of trimethyltin chloride (TMTC). Toxicologist 4, 33.

RUPPERT, P. H., WALSH, T. J., REITER, L. W., AND DYER, R. S. (1982). Trimethyltin-induced hyperactivity: Time course and pattern. Neurobehav. Toxicol. Teratol. 4(2), 135-139.
SCHOENFELd, P. Y., AND HUMPHREYS, M. H. (1976). A general description of the uremic state. In The Kidney, (B. M. Brenner and F. C. Rector, Eds.), Vol. II, pp. 1423-1448. Saunders, Philadelphia.

SiasSi, F., WaNG, M., KoPPlE, J. D., AND SwENDSEID, M. E. (1977). Plasma tryptophan levels and brain serotonin metabolism in chronically uremic rats. $J$. Nutr. 107, 840-845.

Swartzwelder, H. S., Dyer, R. S., Holahan, W., AND MYERS, R. D. (1981). Activity changes in rats following acute trimethyltin exposure. Neurotoxicology 2, 589-593.

Swartzwelder, H. S., Hepler, W., Holahan, W., King, S. E., Leverenz, H. A., Miller, P. A., AND MYER, R. D. (1982). Impaired maze performance in the rat caused by trimethyltin treatment: Problem solving deficits and preservation. Neurobehav. Toxicol. Teratol. 4, 169-176.

Swartzwelder, H. S., Holahan, W., and Myers, R. D. (1983). Antagonism by D-amphetamine of trimethyltin induced hyperactivity: Evidence toward an animal model of hyperkinetic behavior. Neuropharmacology 22, 1049-1054.

WALSH, T. J., MilleR, D. B., AND DYER, R. S. (1982). Trimethyltin, a selective limbic system neurotoxicant, impairs radial-arm maze performance, Neurobehav. Toxicol. Teratol. 4, 177-183.

WeINBERG, J. M., JoHNSON, K. J., DE LA IgLeSIA, F. A., AND ALLEN, E. M. (1987). Acute alterations of tissue $\mathrm{Ca}^{++}$and lethal tubular cell injury during $\mathrm{HgCl}_{2}$ nephrotoxicity in the rat. Submitted. 